\title{
Contributions of traditional and HIV-related risk factors on non-AIDS-defining cancer, myocardial infarction, and end-stage liver and renal diseases in adults with HIV in the USA and Canada: a collaboration of cohort studies
}

\author{
Keri N Althoff, Kelly A Gebo, Richard D Moore, Cynthia M Boyd, Amy C Justice, Cherise Wong, Gregory M Lucas, Marina B Klein, Mari M Kitahata, \\ Heidi Crane, Michael J Silverberg, M John Gill, William Christopher Mathews, Robert Dubrow, Michael A Horberg, Charles S Rabkin, Daniel B Klein, \\ Vincent Lo Re, Timothy R Sterling, Fidel A Desir, Kenneth Lichtenstein, James Willig, Anita R Rachlis, Gregory D Kirk, Kathryn Anastos, \\ FrankJ Palella Jr, Jennifer E Thorne, Joseph Eron, Lisa P Jacobson, Sonia Napravnik, Chad Achenbach, Angel M Mayor, Pragna Patel, Kate Buchacz, \\ Yuezhou Jing, Stephen J Gange for the North American AIDS Cohort Collaboration on Research and Design
}

\section{Summary}

Background Adults with HIV have an increased burden of non-AIDS-defining cancers, myocardial infarction, end-stage liver disease, and end-stage renal disease. The objective of this study was to estimate the population attributable fractions (PAFs) of preventable or modifiable HIV-related and traditional risk factors for non-AIDS-defining cancers, myocardial infarction, end-stage liver disease, and end-stage renal disease outcomes.

Methods We included participants receiving care in academic and community-based outpatient HIV clinical cohorts in the USA and Canada from Jan 1, 2000, to Dec 31, 2014, who contributed to the North American AIDS Cohort Collaboration on Research and Design and who had validated non-AIDS-defining $c$ ancers, $m$ yocardial i nfarction, e nd-stage $l$ iver disease, or end-stage renal disease outcomes. Traditional risk factors were tobacco smoking, hypertension, elevated total cholesterol, type 2 diabetes, renal impairment (stage 4 chronic kidney disease), and hepatitis $C$ virus and hepatitis B virus infections. HIV-related risk factors were low CD4 count $(<200$ cells per $\mu \mathrm{L})$, detectable plasma HIV RNA ( $>400$ copies per $\mathrm{mL}$ ), and history of a clinical AIDS diagnosis. PAFs and 95\% CIs were estimated to quantify the proportion of outcomes that could be avoided if the risk factor was prevented.

Findings In each of the study populations for the four outcomes (1405 of 61500 had non-AIDS-defining cancer, 347 of 29515 had myocardial infarctions, 387 of 35044 had end-stage liver disease events, and 255 of 35620 had end-stage renal disease events), about $17 \%$ were older than 50 years at study entry, about $50 \%$ were non-white, and about $80 \%$ were men. Preventing smoking would avoid 24\% (95\% CI 13-35) of these cancers and 37\% (7-66) of the myocardial infarctions Preventing elevated total cholesterol and hypertension would avoid the greatest proportion of myocardial infarctions: $44 \%(30-58)$ for cholesterol and $42 \%(28-56)$ for hypertension. For liver disease, the PAF was greatest for hepatitis C infection (33\%; 95\% CI 17-48). For renal disease, the PAF was greatest for hypertension (39\%; 26-51) followed by elevated total cholesterol (22\%; 13-31), detectable HIV RNA (19; 9-31), and low CD4 cell count (13\%; 4-21).

Interpretation The substantial proportion of non-AIDS-defining cancers, myocardial infarction, end-stage liver disease, and end-stage renal disease outcomes that could be prevented with interventions on traditional risk factors elevates the importance of screening for these risk factors, improving the effectiveness of prevention (or modification) of these risk factors, and creating sustainable care models to implement such interventions during the decades of life of adults living with HIV who are receiving care.

Funding National Institutes of Health, US Centers for Disease Control and Prevention, the US Agency for Healthcare Research and Quality, the US Health Resources and Services Administration, the Canadian Institutes of Health Research, the Ontario Ministry of Health and Long Term Care, and the Government of Alberta.

\section{Introduction}

Adults receiving antiretroviral therapy (ART) and ageing with HIV have a greater burden of chronic noncommunicable diseases than do adults without HIV. ${ }^{1}$ The increased risk is hypothesised to be the result of a combination of factors, such as the increased prevalence of traditional risk factors for non-communicable diseases in adults with HIV;, ${ }^{2,3}$ the possible synergistic effect of viral coinfection, particularly with hepatitis C virus; ${ }^{4} \mathrm{HIV}$ associated immunosuppression, immune activation, inflammation, and hypercoaguability ${ }^{5}$ (which is blunted but not normalised with ART); ${ }^{6}$ and ART drugs, including previous exposure to first-generation nucleoside analogue reverse transcriptase inhibitors and protease inhibitors.?
Lancet HIV 2019; 6: e93-104 Published Online January 22, 2019 http://dx.doi.org/10.1016 S2352-3018(18)30295-9 This online publication has been corrected. The corrected version first appeared at thelancet.com on April 2, 2019 See Comment page e69 Department of Epidemiology, Johns Hopkins Bloomberg School of Public Health (K N Althoff PhD, C Wong PhD, FA Desir PhD, G D Kirk MD LP Jacobson SCD, Y Jing,

SJ Gange) and Johns Hopkins School of Medicine (K A Gebo MD, R D Moore MD CBoyd MD, G M Lucas MD, J EThorne MD), Johns Hopkins University, Baltimore, MD, USA; Yale School of Medicine, New Haven, CT, USA (A C Justice MD); Veterans Affairs Connecticut Healthcare System, West Haven, CT, USA (A C Justice); McGill University, Montreal, QC, Canada, USA (M B Klein MD); University of Washington, Seattle, WA, USA (M M Kitahata MD, H Crane MD); Kaiser Permanente Northern California, Oakland, CA, USA (M) Silverberg PhD, D B Klein MD); University of Calgary, Calgary, AB, Canada (M J Gill MB); University of California San Diego, San Diego, CA, USA (W C Mathews MD); Yale School of Public Health, New Haven, CT, USA (R Dubrow MD); Kaiser Permanente Mid-Atlantic Permanente Medical Group Rockville, MD, USA (M A Horberg MD); National Cancer Institute, Bethesda, MD, USA (CS Rabkin MD); University of Pennsylvania, Philadelphia, PA, USA (V Lo Re MD); 


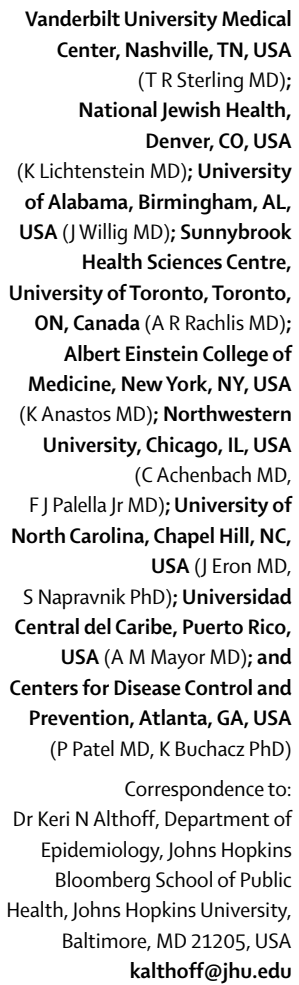

For a map of NA-ACCORD sites see http://www.naaccord.org
Research in context

\section{Evidence before this study}

We searched PubMed with the terms "HIV and cancer", "HIV and myocardial infarction", "HIV and end stage liver disease", and "HIV and end stage renal disease" for articles published in English up to Jan 12, 2018. Studies have shown there is an increased burden of non-AIDS defining cancers, myocardial infarction, end-stage renal disease, and end-stage liver disease among adults ageing with HIV compared with those without HIV. Although both traditional and HIV-related risk factors have been associated with these outcomes, the proportion of outcomes attributed to traditional and HIV-related risk factors can help guide programmes and policies for interventions.

\section{Added value of this study}

To our knowledge, this is the first study to estimate

population-attributable fractions for four age-related

Although some specific antiretroviral drugs have been associated with an increased risk for some noncommunicable diseases, initiation of ART at higher CD4 cell counts results in a decreased risk of cancer in adults ageing with HIV, demonstrating the potential importance of early ART initiation for prevention of non-communicable diseases. ${ }^{8}$ Despite known roles of traditional and HIV-related risk factors in the development of non-communicable diseases among adults with HIV, how much of the burden can be attributed to each of these factors is unknown.

Over the past two decades, cancer, cardiovascular disease, and liver disease have become the top causes of non-AIDS-related death among adults with HIV., ${ }^{910}$ Endstage liver disease is also responsible for substantial morbidity among adults with HIV because of the high prevalence of chronic hepatitis B and hepatitis $\mathrm{C}$ infection in this population. ${ }^{11-13}$ Although rare, end-stage renal disease is more common among people with HIV than among the general population ${ }^{14}$ and has been hypothesised to increase as a result of the combined effect of nephrotoxic ART regimens and age-associated declines in renal function..$^{15}$

Our goal was to estimate the proportion of non-AIDSdefining cancers, myocardial infarction, end-stage liver disease, and end-stage renal disease events that can be attributed to traditional and HIV-related risk factors among adults with HIV who have been successfully linked into care. To this end, we analysed traditional and HIV-related risk factors for these four validated noncommunicable disease outcomes among participants of the North American AIDS Cohort Collaboration on Research and Design (NA-ACCORD). We used a population attributable fraction (PAF) approach to quantify the proportion of non-communicable diseases that could be eliminated if particular risk factors were not present, and can be used to inform prioritisation of conditions in a large study population of adults with HIV. Risk factors were grouped as traditional or HIV-related to provide direction to programmes and policies for preserving health among adults ageing with HIV.

\section{Implications of all the available evidence}

Traditional risk factors contribute greatly to the burden of age-related diseases in adults with HIV. Early ART initiation, implementing care models that can sustain a focus on traditional risk-factor reduction after ART initiation and viral suppression, and improving the effectiveness of risk factor interventions are essential to preserving health among adults with HIV.

interventions aimed at reducing risk factor exposure to preserve the health of adults with HIV.

\section{Methods \\ Study design and participants}

The NA-ACCORD is the largest consortium of HIV cohorts in the USA and Canada and is the North American region of the International Epidemiologic Databases to Evaluate AIDS (IeDEA) project, supported by the National Institutes of Health. Details on this collaboration have been published previously. ${ }^{16}$ Briefly, the NA-ACCORD consists of single-site and multisite clinical and interval cohorts in various settings, ranging from community health centres to academic institutions. The cohorts have prospectively collected data on over 180000 adults ( $\geq 18$ years) with HIV who had at least two care visits within 12 months from more than 200 sites in the USA and Canada. Each cohort submits comprehensive data on enrolled participants to the Data Management Core (University of Washington, Seattle, WA, USA), where the data undergo quality control, are harmonised across cohorts, and are transmitted to the Epidemiology/ Biostatistics Core (Johns Hopkins University, Baltimore, MD, USA), which did the analyses presented in this Article.

Participants from clinical cohorts within the NA-ACCORD with complete access to both inpatient and outpatient electronic medical records that validated the occurrences of non-AIDS-defining cancers, myocardial infarction, end-stage renal disease, and end-state liver disease were eligible for inclusion in the analyses presented in this Article.

The human-participant research activities of the NA-ACCORD and each of the participating cohort studies have been reviewed and approved by their respective local institutional review boards and the Johns Hopkins School of Medicine. The Johns Hopkins Bloomberg School of 
Public Health internal review board approved the present study.

\section{Procedures}

The protocol for ascertainment and validation of cancer, type 1 myocardial infarction, end-stage liver disease, and end-stage renal disease (outcomes of interest) within the NA-ACCORD has been previously published (appendix pp 1-3). ${ }^{17-19}$ First non-AIDS-defining cancer (which excludes cervical cancer, non-Hodgkin lymphoma, and Kaposi's sarcoma) was our cancer outcome of interest; non-melanoma skin cancers and precancers were also excluded. Only type 1 myocardial infarctions that were the result of atherothrombotic coronary events from plaque rupture were included; type 2 myocardial infarctions have different causes (eg, hypotension, hypoxia, and stimulant-induced spasm resulting in increased oxygen demand or decreased supply).

Calendar periods in which validated outcomes were actively ascertained (observation window) were defined for each outcome and for each cohort. Participants entered our nested studies at enrolment into the NA-ACCORD, the start of the outcome observation window, or Jan 1, 2000, whichever came later. Participants were followed up until the latest of: event of interest; death; 1 year after the date of the last CD4 cell count or HIV RNA measurement; the end of the outcome observation window; or Dec 31, 2009, for end-stage liver and renal outcomes, Dec 31, 2013, for the myocardial infarction outcome, or Dec 31, 2014, for the non-AIDS defining cancer outcome. We excluded prevalent cases of our events of interest.

Traditional risk factors were chosen on the basis of previously identified $r$ isk factors for the o utcomes of interest and data availability, and included cigarette smoking (ever or never), elevated total cholesterol ( $\geq 240 \mathrm{mg} / \mathrm{dL}$; statin prescription was accounted for separately in analyses), hypertension (clinical diagnosis and an antihypertensive medication prescription), type 2 diabetes (diabetes-specific medication, a diagnosis with a diabetes-related medication, or a glycosylated haemoglobin $\geq 6 \cdot 5 \%$ ), stage 4 chronic kidney disease (estimated glomerular filtration $\mathrm{r}$ ate $<30 \mathrm{~m} \mathrm{~L} / \mathrm{min}$ per $1.73 \mathrm{~m}^{2}$ vs $\geq 30 \mathrm{~mL} / \mathrm{min}$ per $1.73 \mathrm{~m}^{2}$, with the Chronic Kidney Disease Epidemiology Collaboration equation $\left.{ }^{20}\right)$. Elevated total cholesterol, hypertension, diabetes, and stage 4 chronic kidney disease were time varying with the restriction that once an individual met the definition, $t$ he c lassification wa $s$ no $t$ re versible.

At-risk alcohol use (ever reporting $\geq 3$ drinks per day or $\geq 7$ drinks per week for women, or $\geq 4$ drinks/day or $\geq 14$ drinks/week for men) and body-mass index measurements were available for a subgroup of participants enrolled in contributing cohorts that measured these risk factors.

We classified h epatitis $\mathrm{C}$ i nfection ( having a p ositive antibody test or detectable RNA, or the presence of a hepatitis $\mathrm{C}$ genotype test; ever vs never) and hepatitis $\mathrm{B}$ infection (having a positive surface-antigen test, a positive e-antigen test, or a detectable DNA test result; ever vs never) as preventable and modifiable traditional risk factors because of their causal relationships with liver cancer and end-stage liver disease.

Traditional HIV-related risk factors included CD4 cell count (categorised using the clinically meaningful cutoff for severe immune deficiency threshold of $<200$ cells per $\mu \mathrm{L}$ versus $\geq 200$ cells per $\mu \mathrm{L}$ ), HIV viral suppression (HIV-1 RNA $\leq 400$ copies per $\mathrm{mL}$ ), and a history of a clinical AIDS-defining illness excluding the criteria of a CD4 count of less than 200 cells per $\mu \mathrm{L}^{21}$ CD4 cell count and HIV viral suppression were time varying. As is true in clinical care, a history of AIDS was also time varying with the restriction that once an individual met the definition, the classification was not reversible. CD4 counts of less than 200 cells per $\mu \mathrm{L}$ and detectable viral loads are modifiable with effective ART, and AIDS is preventable with early HIV diagnosis and ART initiation. ART prescription was not considered as a risk factor because of the associations of ART with CD4 cell count, HIV RNA, and clinical AIDS diagnosis. Investigations of specific antiretroviral drugs, such as abacavir or tenofovir, that would require additional adjustment for confounding by indication are outside the scope of our study.

Non-modifiable covariates included age, sex (male and female), race and ethnicity (white, black, hispanic, and other), and HIV-acquisition risk group (men who have sex with men, individuals reporting current or previous injection drug use, heterosexual, and other) were selfreported at enrolment into the NA-ACCORD. Although current use is a modifiable risk factor, injection drug use as the suspected mode of HIV transmission is not modifiable.

\section{Statistical analysis}

All analyses were done separately for each outcome (non-AIDS-defining cancers, myocardial infarction, endstage liver disease, and end-stage renal disease), and an individual could contribute data to each of the outcomespecific analyses.

As PAFs incorporate the prevalence of risk factors among people with the outcome (estimated as a simple proportion) and the risk of the outcome, we estimated adjusted hazard ratios (aHR) and 95\% CIs with Cox proportional hazard models with piecewise constant baseline hazard functions. The PAFs approach for disease incidence described by Laaksonen ${ }^{22}$ was used because it accommodated time-varying risk factors available from a cohort study design. Death would preclude the outcome of interest. The Laaksonen approach ${ }^{22}$ accounted for mortality as well as the associations between death and the risk factors of interest in the estimation of the PAF. Participant follow-up time was divided into periods of 2 years. Age, sex, race and ethnicity, and injection drug
See Online for appendix 
use status were accounted for in adjusted models that produced PAF estimates and 95\% CIs. Schoenfeld residual global tests and Kolmogorov-type supremum tests were used to test the a ssumption of proportional hazards for all risk factors of interest. ${ }^{23}$
A sensitivity analysis was done after removing lung cancer from the non-AIDS-defining cancers outcome as it was the most common cancer and has a strong relationship with smoking. A sensitivity analysis was also done after removing elevated total cholesterol from the end-stage

\begin{tabular}{|c|c|c|c|c|c|c|c|c|}
\hline & \multicolumn{2}{|l|}{ NADC } & \multicolumn{2}{|l|}{ MI } & \multicolumn{2}{|l|}{ ESLD } & \multicolumn{2}{|l|}{ ESRD } \\
\hline & $\begin{array}{l}\text { No diagnosis } \\
(\mathrm{n}=60095)\end{array}$ & $\begin{array}{l}\text { Diagnosis } \\
(n=1405)\end{array}$ & $\begin{array}{l}\text { No diagnosis } \\
(\mathrm{n}=29168)\end{array}$ & $\begin{array}{l}\text { Diagnosis } \\
(n=347)\end{array}$ & $\begin{array}{l}\text { No diagnosis } \\
(\mathrm{n}=34657)\end{array}$ & $\begin{array}{l}\text { Diagnosis } \\
(n=387)\end{array}$ & $\begin{array}{l}\text { No diagnosis } \\
(\mathrm{n}=35365)\end{array}$ & $\begin{array}{l}\text { Diagnosis } \\
(n=255)\end{array}$ \\
\hline \multicolumn{9}{|l|}{ Demographics } \\
\hline$<40$ years & $29429(49 \%)$ & $317(23 \%)$ & $13749(47 \%)$ & $63(18 \%)$ & $16641(48 \%)$ & $124(32 \%)$ & $16344(46 \%)$ & $94(37 \%)$ \\
\hline 40-49years & $20584(34 \%)$ & $550(39 \%)$ & $10217(35 \%)$ & $144(41 \%)$ & $12474(36 \%)$ & $160(41 \%)$ & $13042(37 \%)$ & $101(40 \%)$ \\
\hline 50-59 years & 8239 (14\%) & $390(28 \%)$ & $4220(14 \%)$ & $106(31 \%)$ & $4528(13 \%)$ & $80(21 \%)$ & 4835 (14\%) & $41(16 \%)$ \\
\hline Male & $46330(77 \%)$ & $1093(78 \%)$ & $23475(80 \%)$ & $298(86 \%)$ & $27354(79 \%)$ & $334(86 \%)$ & $27974(79 \%)$ & $178(70 \%)$ \\
\hline \multicolumn{9}{|l|}{ Race and ethnicity } \\
\hline White & $25075(42 \%)$ & $692(49 \%)$ & $13429(46 \%)$ & $193(56 \%)$ & $14560(42 \%)$ & $207(53 \%)$ & $15022(42 \%)$ & $30(12 \%)$ \\
\hline Black & $21658(36 \%)$ & $534(38 \%)$ & $10831(37 \%)$ & $123(35 \%)$ & $11693(34 \%)$ & $108(28 \%)$ & $11452(32 \%)$ & $205(80 \%)$ \\
\hline Hispanic & $7683(13 \%)$ & $111(8 \%)$ & 3106 (11\%) & $20(6 \%)$ & $4379(13 \%)$ & $43(11 \%)$ & $4756(13 \%)$ & $12(5 \%)$ \\
\hline Other & $3033(5 \%)$ & $44(3 \%)$ & $1308(4 \%)$ & $10(3 \%)$ & $1269(4 \%)$ & $7(2 \%)$ & $1333(4 \%)$ & $1(0 \%)$ \\
\hline IDU & $6885(11 \%)$ & $204(15 \%)$ & $2971(10 \%)$ & $44(13 \%)$ & $4231(12 \%)$ & $97(25 \%)$ & $3912(11 \%)$ & $52(20 \%)$ \\
\hline Heterosexual contact & $15397(26 \%)$ & $343(24 \%)$ & $7559(26 \%)$ & $84(24 \%)$ & $9224(27 \%)$ & $73(19 \%)$ & $9065(26 \%)$ & $108(42 \%)$ \\
\hline Other, unknown, or missing & $6443(11 \%)$ & $116(8 \%)$ & $2535(9 \%)$ & $26(7 \%)$ & $3688(11 \%)$ & $37(10 \%)$ & $5274(15 \%)$ & $28(11 \%)$ \\
\hline \multicolumn{9}{|l|}{ Traditional Risk Factors } \\
\hline \multicolumn{9}{|l|}{ Smoking } \\
\hline Never & $13604(23 \%)$ & $236(17 \%)$ & $6891(24 \%)$ & $55(16 \%)$ & $6916(20 \%)$ & $57(15 \%)$ & $7645(22 \%)$ & $41(16 \%)$ \\
\hline Ever & $37013(62 \%)$ & 1106 (79\%) & $21884(75 \%)$ & $292(84 \%)$ & 20588 (59\%) & $289(75 \%)$ & $20711(59 \%)$ & $182(71 \%)$ \\
\hline Missing & $9478(16 \%)$ & $63(4 \%)$ & $393(1 \%)$ & $0(0 \%)$ & $7153(21 \%)$ & $41(11 \%)$ & $7009(20 \%)$ & $32(13 \%)$ \\
\hline Elevated TC & $10037(17 \%)$ & $325(23 \%)$ & $7287(25 \%)$ & $211(61 \%)$ & $7032(20 \%)$ & $66(17 \%)$ & $6779(19 \%)$ & $108(42 \%)$ \\
\hline Hypertension & $5230(9 \%)$ & $237(17 \%)$ & $2809(10 \%)$ & $89(26 \%)$ & $2234(6 \%)$ & $53(14 \%)$ & $2365(7 \%)$ & $68(27 \%)$ \\
\hline Diabetes & $1660(3 \%)$ & $68(5 \%)$ & $766(3 \%)$ & $27(8 \%)$ & $855(2 \%)$ & $20(5 \%)$ & $928(3 \%)$ & $29(11 \%)$ \\
\hline \multicolumn{9}{|l|}{ ART regimen } \\
\hline Treatment naive & $25216(42 \%)$ & $452(32 \%)$ & $11478(39 \%)$ & $90(26 \%)$ & $14170(41 \%)$ & $96(25 \%)$ & $13065(37 \%)$ & $75(29 \%)$ \\
\hline PI-based ART & $12394(21 \%)$ & $406(29 \%)$ & $5842(20 \%)$ & $114(33 \%)$ & $7707(22 \%)$ & $117(30 \%)$ & $7606(22 \%)$ & $51(20 \%)$ \\
\hline NNRTI-based ART & $10289(17 \%)$ & $217(15 \%)$ & $5121(18 \%)$ & $44(13 \%)$ & $5465(16 \%)$ & $50(13 \%)$ & $6756(19 \%)$ & $35(14 \%)$ \\
\hline Other ART & $5591(9 \%)$ & $155(11 \%)$ & $2402(8 \%)$ & $43(12 \%)$ & $2858(8 \%)$ & $56(14 \%)$ & $3278(9 \%)$ & $35(14 \%)$ \\
\hline $\begin{array}{l}\text { Treatment experienced but } \\
\text { not prescribed ART }\end{array}$ & $6558(11 \%)$ & $174(12 \%)$ & $4325(15 \%)$ & $56(16 \%)$ & $4457(13 \%)$ & $68(18 \%)$ & $4660(13 \%)$ & $59(23 \%)$ \\
\hline
\end{tabular}

Values are $\mathrm{n}(\%)$. NADC=non-AIDS-defining cancers. $M I=$ myocardial infarction. ESLD=end-stage liver disease. ESRD=end-stage renal disease. MSM=men who have sex with men. IDU=history of injection drug use. $T C=$ total cholesterol. $C K D=$ chnic kidney disease. $\mathrm{HCV}=$ hepatitis $\mathrm{C}$ virus. $\mathrm{HBV}=$ hepatitis $\mathrm{B}$ virus. $\mathrm{ART}=$ antiretroviral therapy. $\mathrm{Pl}=$ protease inhibitor. $\mathrm{NNRTI=non-nucleoside} \mathrm{reverse} \mathrm{transcriptase} \mathrm{inhibitor.}$

Table: Participant characteristics at study entry, by non-AIDS-defining cancers ( $n=61500)$, myocardial infarction ( $n=29515)$, end-stage liver disease ( $n=35044)$, and end-stage renal disease $(n=35620)$ diagnoses 
renal disease outcome because of the relationship of renal impairment and dyslipidaemia. ${ }^{24}$ Subgroup analyses were done for the myocardial infarction outcome among people with body mass-index data and for the end-stage liver disease outcome among people with at-risk alcohol use data; cohorts that did not contribute data on these risk factors were excluded from the subgroup analyses. All analyses were done using SAS version 9.3; statistical interpretation was guided by a $\mathrm{p}$ value of less than 0.05 and PAF $95 \%$ CIs that do not cross $0 \%$.

\section{Role of the funding source}

The funders of the study had no role in the study design, data collection, data analysis, data interpretation, or writing of the manuscript. The content is solely the responsibility of the authors and does not necessarily represent the official views of the funders. YJ had full access to all the data in the study and KNA had final responsibility for the decision to submit for publication.

\section{Results}

Among 72854 NA-ACCORD participants from 21 cohorts with validated non-AIDS-defining cancer outcomes, 9202 were excluded as prevalent cases or not contributing observation time during the study period (Jan 1, 2000, to Dec 31, 2014) and 2152 were excluded for having an AIDS-defining cancer as their first cancer, resulting in 61500 participants, of whom 1405 (2\%) had a validated non-AIDS defining cancer diagnosis. Among 34708 participants from seven cohorts with validated myocardial infarction diagnoses, 5193 were excluded as prevalent cases or not contributing observation time during the study period (Jan 1, 2000, to Dec 31, 2013), resulting in 29515 participants, of whom 347 (1\%) had a validated myocardial infarction diagnosis. Among 42609 participants from 12 cohorts with validated endstage liver disease diagnoses, 7025 were excluded as prevalent cases or not contributing observation time during the study period (Jan 1, 2000, to Dec 31, 2009),

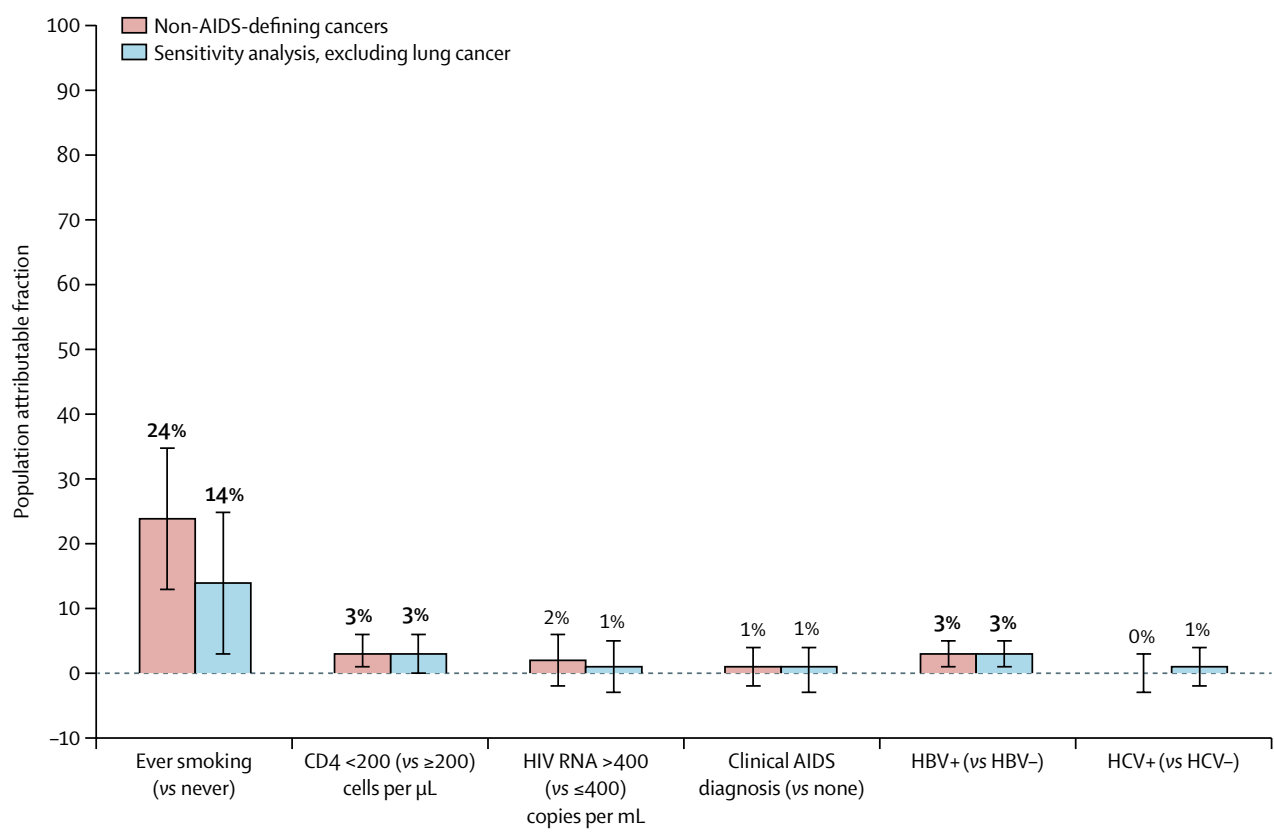

Non-AIDS-defining cancers $(n=61500)$ Prevalence

$95 \% \mathrm{Cl})$

Sensitivity analysis: non-AIDSdefining cancers, excluding lung cancer $(n=61235)$

$$
\begin{array}{r}
\text { Prevalence } \\
\text { aHR } \\
(95 \% \mathrm{Cl})
\end{array}
$$

$$
\begin{gathered}
79 \% \\
\mathbf{1 . 6 1} \\
\mathbf{( 1 . 3 7 - 1 . 8 9 )}
\end{gathered}
$$

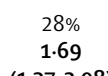

$(1 \cdot 37-2 \cdot 08)$
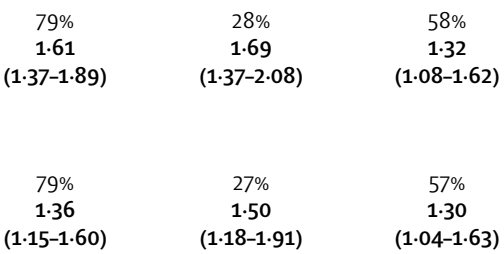
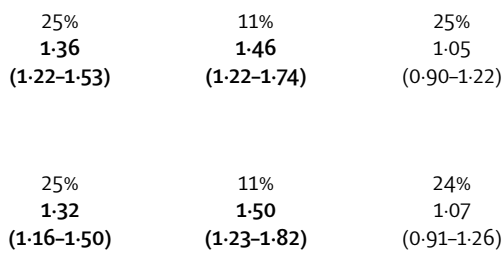

$1 \cdot 32$
$(1 \cdot 16-1 \cdot 50)$

$(0.90-1.22$

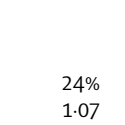

$(0.91-1 \cdot 26)$ 
resulting in 35044 participants, of whom 387 (1\%) had a validated end-stage liver disease diagnosis. Among 43961 participants from 12 cohorts with validated endstage renal disease diagnoses, 8341 were excluded as prevalent cases or not contributing observation time during the study period (Jan 1, 2000, to Dec 31, 2009), resulting in 35620 participants, of whom 255 (1\%) had a validated end-stage liver disease diagnosis (appendix p 4).

Median follow-up time was similar across the four outcomes: 3.7 years (IQR 1.4-7.7) for non-AIDSdefining cancer, $3 \cdot 5$ years $(1 \cdot 5-6 \cdot 9)$ for myocardial infarction, $3 \cdot 1$ years $(1 \cdot 3-6 \cdot 2)$ for end-stage liver disease, and 3.3 years $(1.4-5 \cdot 7)$ for end-stage renal disease (appendix, p 5). Of the 1405 non-AIDS-defining cancers observed, 230 (16\%) were lung, 225 (16\%) were anal, 167 (12\%) were prostate, 96 (7\%) were Hodgkin lymphoma, $90(6 \%)$ were liver, $83(6 \%)$ were oral cavity and pharynx, $82(6 \%)$ were breast, $69(5 \%)$ were melanoma, and $65(5 \%)$ were colon and rectum cancers. Each of the other type-specific cancers accounted for less than $5 \%$ of the 1405 cancer diagnoses (appendix p 6).

For all four incident outcomes, there was a greater proportion of participants with a smoking history, hepatitis C and B infection, low CD4 cell count $(<200$ cells per $\mu \mathrm{L})$, and a history of a clinical AIDS diagnosis among individuals with an incident outcome than among those who did not develop the outcomes (table). The prevalence of elevated total cholesterol, hypertension, diabetes, and stage 4 chronic kidney disease was higher among individuals with an incident myocardial infarction than among those without. The proportion of individuals with a detectable viral load was higher among those with a diagnosis of end-stage renal disease than among those without an incident diagnosis, but the proportion of individuals with detectable HIV RNA was similar by incident diagnosis status for all other outcomes. Individuals with an incident diagnosis of end-stage renal diseases also had

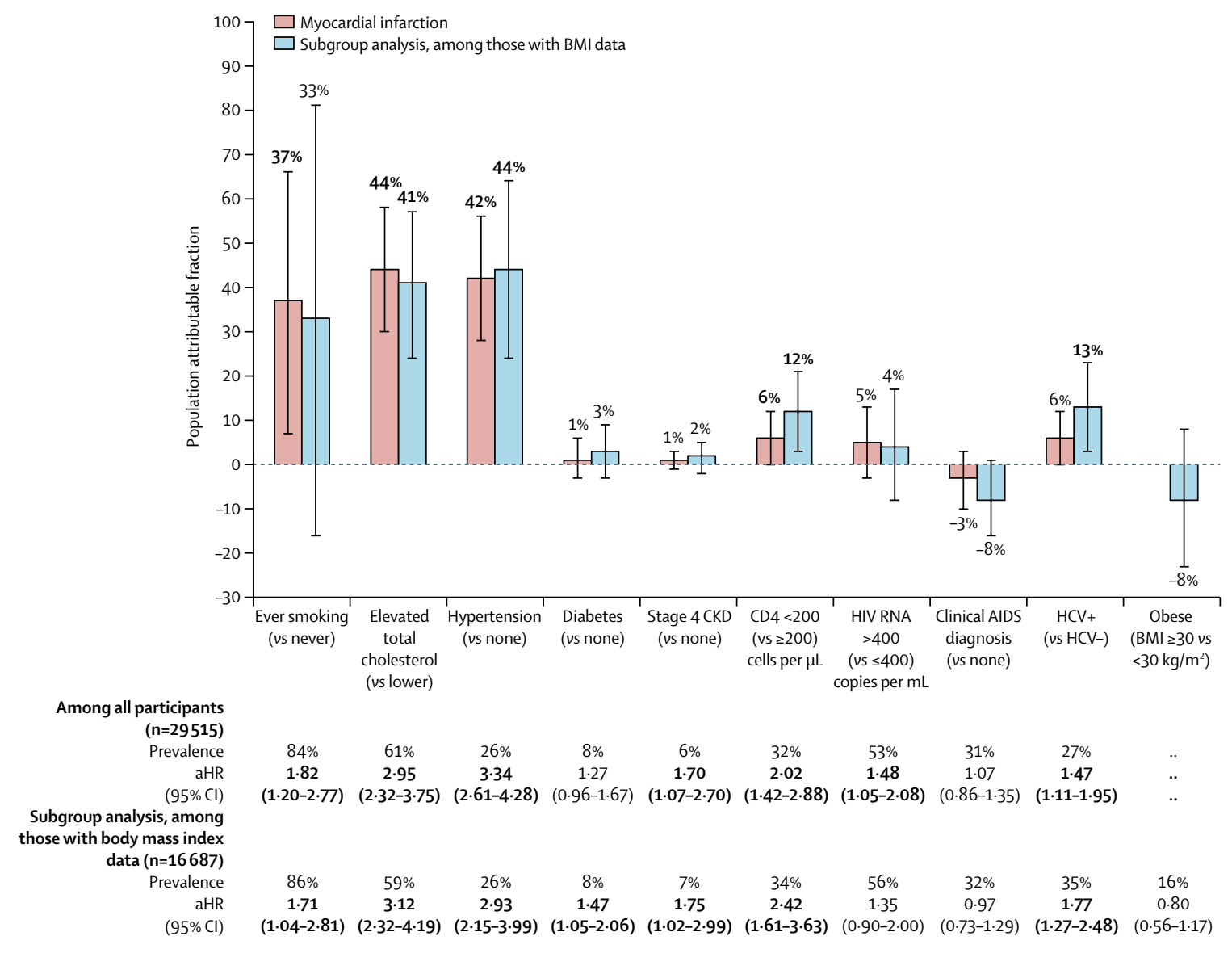

Figure 2: Population attributable fractions for traditional and HIV-related risk factors for myocardial infarction, overall $(n=29515)$ and among those with BM data $(n=16687)$

Bars indicate $95 \% \mathrm{Cl}$. The calculation of the population attributable fraction allows for time-varying risk factors. The subgroup analysis was restricted to the 16687 (57\%) participants for whom BMI data were available; 227 of these participants had a type 1 myocardial infarction diagnosis while under observation and 16460 did not. Below the plot, prevalence is the prevalence of the risk factor among those with the outcome at study entry; aHRs were adjusted for age, sex, race and ethnicity, history of injection drug use, and all the risk factors shown in the figure. Bold indicates significant estimates. aHR=adjusted hazard ratio. $\mathrm{BMI}=$ body-mass index. $\mathrm{HBV}=$ hepatitis $\mathrm{B}$ virus infection. $\mathrm{HCV}=$ hepatitis C virus infection. 

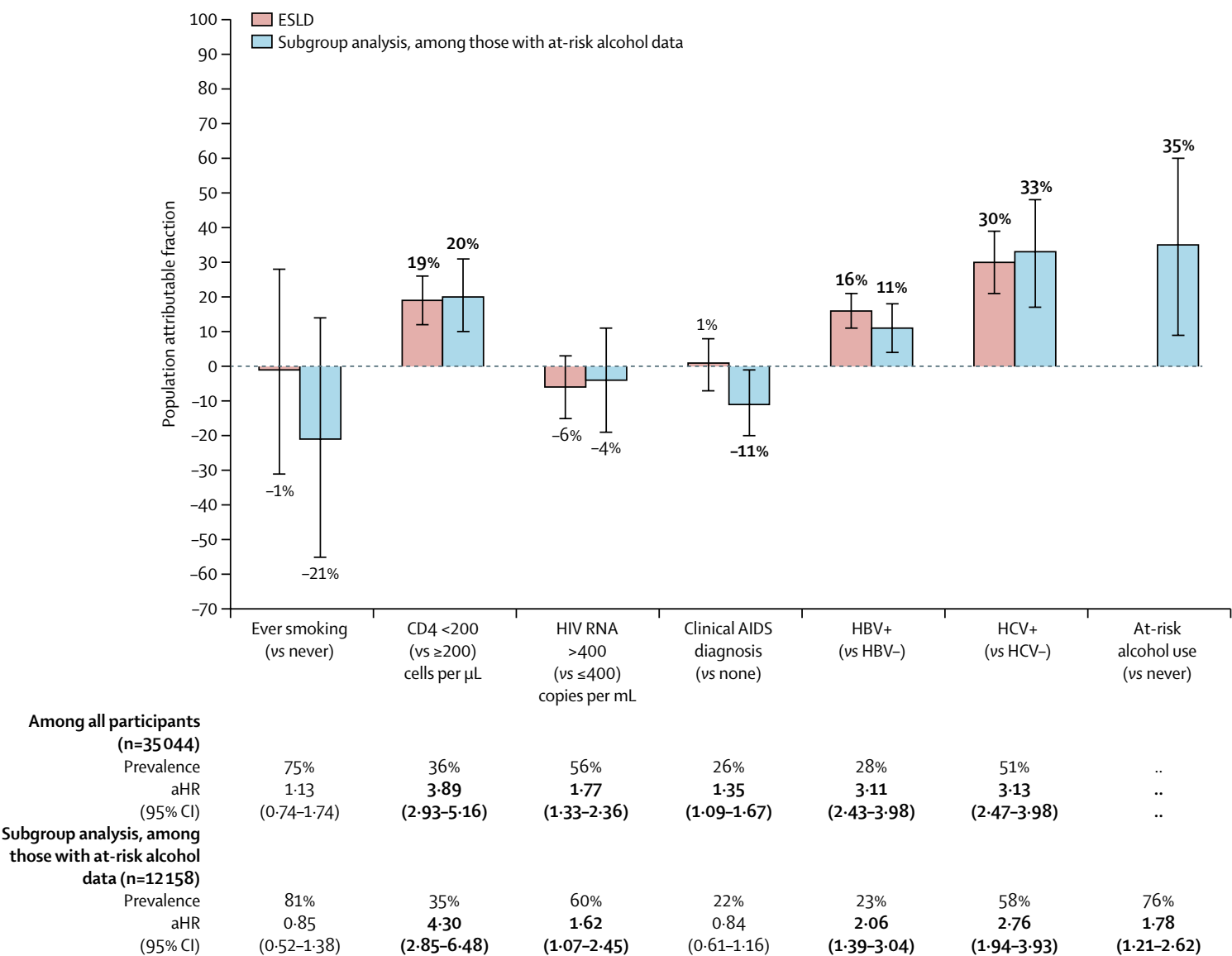

$$
\begin{gathered}
75 \% \\
1 \cdot 13 \\
(0 \cdot 74-1 \cdot 74)
\end{gathered}
$$
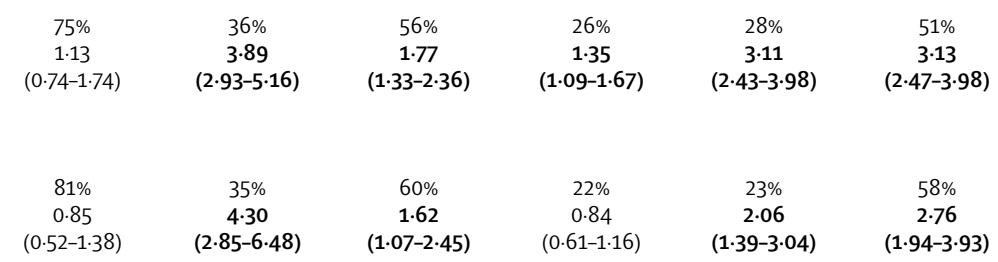

$35 \%$
$4 \cdot 30$
$(2 \cdot 85-6 \cdot 48)$

$60 \%$
$1 \cdot 62$
$(1 \cdot 07-2 \cdot 45)$

$22 \%$
$0 \cdot 84$
$(0 \cdot 61-1 \cdot 16)$

$23 \%$
$\mathbf{2} \cdot 06$
$(\mathbf{1} \cdot 39-3 \cdot 04)$

$58 \%$
$2 \cdot 76$
$(\mathbf{1} \cdot 94-3 \cdot 93)$

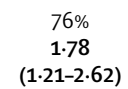

Figure 3: Population attributable fractions for traditional and HIV-related risk factors for ESLD, overall $(n=35044)$ and among those with at-risk alcohol use data $(n=12158)$

Bars indicate $95 \% \mathrm{Cl}$. The calculation of the population attributable fraction allows for time-varying risk factors. The subgroup analysis was restricted to the 12158 (35\%) participants for whom at-risk alcohol-use data were available; 176 of these participants had ESLD diagnosis while under observation and 11982 did not. Below the plot, prevalence is the prevalence of the risk factor among those with the outcome at study entry; aHRs were adjusted for age, sex, race and ethnicity, history of injection drug use, and all the risk factors shown in the figure. Bold indicates significant estimates. aHR=adjusted hazard ratio. BMI=body-mass index. $E S L D=e n d$-stage liver disease. $\mathrm{HBV}=$ hepatitis $B$ virus infection. $\mathrm{HCV}=$ hepatitis C virus infection.

greater proportions of elevated total cholesterol, hypertension, and diabetes than those who did not develop this outcome.

In addition to the prevalence of the risk factor, PAFs are influenced by the risk of the outcome associated with each preventable or modifiable risk factor. We calculated the aHRs allowing some risk factors (ie, elevated total cholesterol, hypertension, type 2 diabetes, stage 4 chronic kidney disease, low CD4 cell count, detectable HIV RNA, history of AIDS-defining illness) to vary with time and adjusting for age (time varying), sex, race and ethnicity, and a history of injection drug use.

For non-AIDS-defining $\mathrm{c}$ ancer, $\mathrm{t}$ he $\mathrm{p}$ reventable o $\mathrm{r}$ modifiable $\mathrm{r}$ isk $\mathrm{f}$ actors $\mathrm{w}$ ith $\mathrm{s}$ ignificant as sociations were smoking, low CD4 cell count, detectable HIV RNA, a history of clinical AIDS diagnosis, and hepatitis B infection (figure 1). In a sensitivity analysis excluding individuals with lung cancer diagnoses, the effect of smoking attenuated, but there was still an association between smoking and non-AIDS-defining cancer (aHR 1·36, 95\% CI 1·15-1·60).

Smoking, elevated total cholesterol, hypertension, stage 4 chronic kidney disease, a low CD4 cell count, detectable HIV RNA, and hepatitis C infection were associated with myocardial infarction (figure 2). In a subgroup analysis restricted to 16687 individuals (57\%) with available body mass-index measurements, there was no evidence of an association of obesity with myocardial infarction in the adjusted model, whereas the associations of elevated total cholesterol, diabetes, stage 4 chronic kidney disease, low CD4 cell count, and hepatitis $\mathrm{C}$ infection with this outcome strengthened; all other associations attenuated.

For end-stage liver disease, low CD4 cell count, detectable HIV RNA, a history of a clinical AIDS diagnosis, and hepatitis infection (B or C) were associated with the outcome (figure 3). In a subgroup analysis restricted to 12158 participants (35\%) with known at-risk alcohol 
status, at-risk alcohol consumption was associated with end-stage liver disease. The inclusion of at-risk alcohol consumption in the model diminished the effects of smoking and a history of a clinical AIDS diagnosis, strengthened the association with low CD4 cell count, and attenuated the effects of the remaining risk factors (figure 3).

For end-stage renal disease, elevated total cholesterol, hypertension, diabetes, low CD4 cell count, detectable HIV RNA, and a history of clinical AIDS diagnosis showed significant associations (figure 4).

Schoenfeld residual global test approach and the Kolmogorov-type supremum test of the proportional hazards assumption showed proportionality for all risk factors presented (data not shown).

The PAF for smoking was substantial for non-AIDSdefining cancer $(24 \%, 95 \%$ CI $13-35)$ and myocardial infarction (37\%, 7-66), but was not for end-stage liver or renal disease. For the non-AIDS-defining cancers outcome, the PAF for smoking was greater the PAFs for HIV-related risk factors and persisted after excluding lung cancer cases.

For myocardial infarction, PAFs were higher for elevated total cholesterol (44\%, 95\% CI 30-58) and hypertension $(42 \%, 28-56)$ than for smoking (37\%, 7-66); these were all larger than those for HIV-related risk factors. In the subgroup analysis among persons with body mass-index measurements, after adjustment for body-mass index, the magnitude of the PAFs for traditional risk factors did not meaningfully change (however, the PAF for smoking was no longer significant), and the magnitude of the PAFs for the low CD4 cell count and hepatitis $\mathrm{C}$ infection increased slightly (and the PAF for hepatitis $\mathrm{C}$ infection became significant).

Hepatitis C infection had the highest PAF (30\%, 95\% CI 21-39) for end-stage liver disease, followed by low CD4 cell count $(19 \%, 12-26)$ and hepatitis B infection $(16 \%, 11-21)$. In the subgroup analysis among individuals with at-risk alcohol consumption measurements, the PAF for at-risk alcohol consumption $(35 \%, 9-60)$ was greater than that for hepatitis C infection (33\%, 17-48).

Hypertension had the highest PAF for end-stage renal disease (39\%, 95\% CI 26-51). PAFs were similar for elevated total cholesterol $(22 \%, 13-31)$ and detectable HIV RNA (19\%, 6-31), whereas we estimated lower PAFs for low CD4 cell count (13\%, 4-21) and diabetes $(6 \%$, 1-10). We found no significant contribution for smoking, a history of a clinical AIDS diagnosis, or hepatitis C infection to the increased burden of end-stage renal disease. In the sensitivity analysis, the PAFs for the remaining risk factors did not vary substantially after removal of total elevated cholesterol (data not shown).

PAFs grouped by risk factors (as opposed to oucomes) are shown in the appendix (pp 7-8).

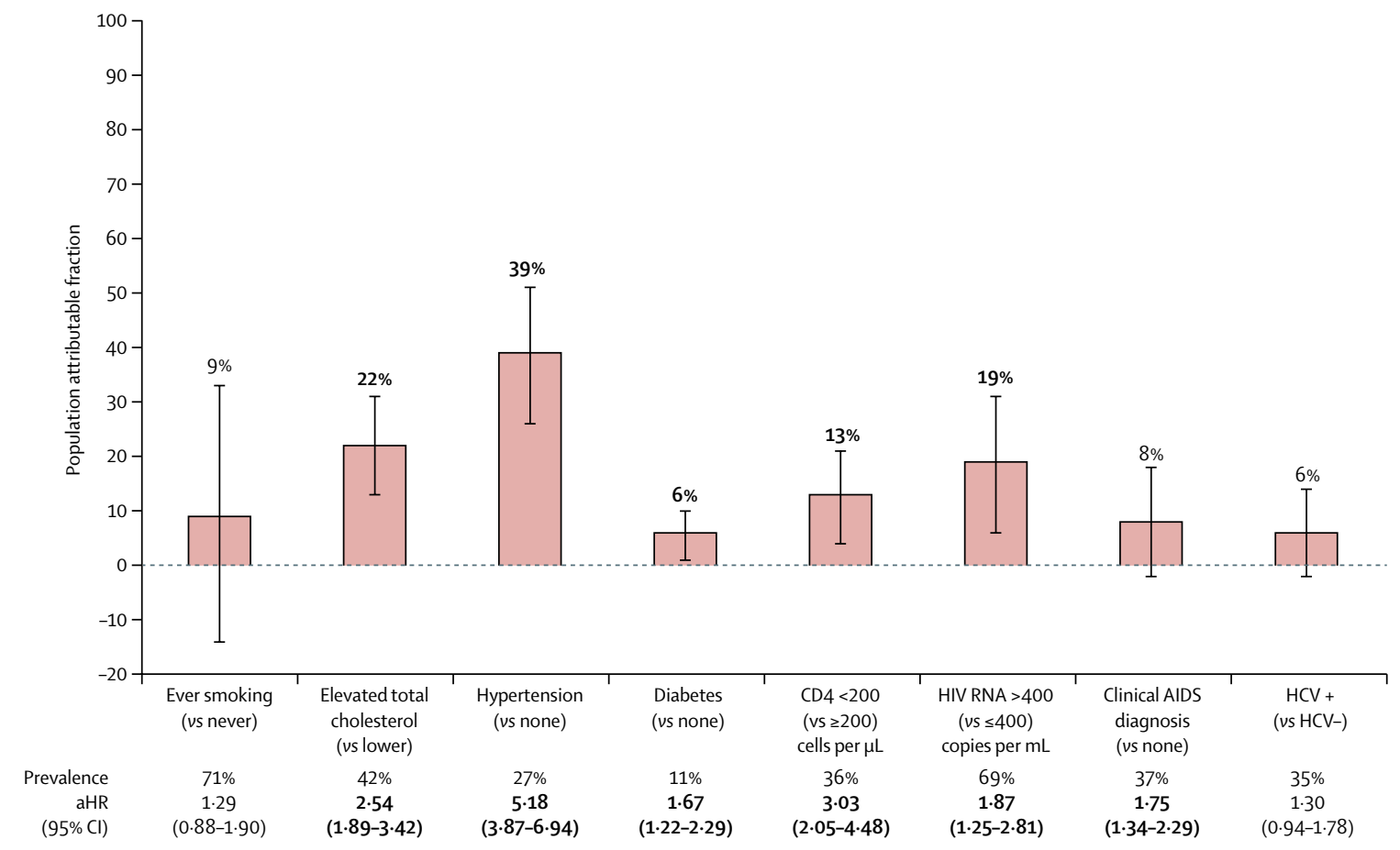

Figure 4: Population attributable fractions for traditional and HIV-related risk factors for ESRD $(n=35620)$

Bars indicate $95 \% \mathrm{Cl}$. The calculation of the population attributable fraction allows for time-varying risk factors. Below the plot, prevalence is the prevalence of the risk factor among those with the outcome at study entry; aHRs were adjusted for age, sex, race and ethnicity, history of injection drug use, and all the risk factors shown in the figure. This analysis of population attributable factors for factors associated with ESRD did not include accounting for stages of chronic kidney disease. Bold indicates statistically significant estimate. aHR=adjusted hazard ratio. ESRD=end-stage renal disease. $H C V=$ hepatitis C virus infection. 


\section{Discussion}

In the population of adults with HIV who were successfully linked to care, we found that traditional risk factors, including smoking, elevated total cholesterol, hypertension, and chronic hepatitis $\mathrm{C}$ infection accounted for a larger share of the non-AIDS-defining cancer, myocardial infarction, end-stage liver disease, and end-stage renal disease incident outcomes than did HIV-related risk factors. The PAF approach accounts for not only the risk of the outcome associated with the risk factor, but also the prevalence of the risk factor among individuals with the outcome. A high PAF can result from a risk factor with a weak or moderately strong association with the outcome but with a high prevalence; conversely, a low PAF can result from a strong risk factor with a low prevalence. With both pieces of information, the evidence from our study is clear: to avoid sizeable proportions of non-AIDS-defining $\mathrm{c}$ ancers, $\mathrm{m}$ yocardial infarction, end-stage liver disease, and end-stage renal disease, the continued focus on maintaining HIV viral suppression after ART initiation must be balanced with screening for traditional risk factors, effective interventions to reduce the burden of traditional risk factors, and a sustainable model of care with the capacity to provide traditional risk factor interventions over the decades of life with HIV.

Many interventions used to reduce traditional risk factor burden in adults with HIV were developed in the general population and might need modifications to improve effectiveness in adults with HIV. For example, smoking cessation programmes with reduced financial barriers to nicotine replacement therapy and increased social support could improve the scarce success of smoking cessation programmes, a challenge that plagues both adults with and without HIV, but has a greater effect on adults with HIV because of the two-times higher prevalence of smoking in this population than in the general population. ${ }^{25-28}$ Similarly, interventions for alcohol use reduction and for screening and treating dyslipidaemia, hypertension, and diabetes might need tailoring to meet the needs of adults with HIV and to have a substantial effect. Beyond the individual-level interventions, structural and policy interventions on traditional risk factors could have large effects a mong adults with HIV, who carry an increased burden of traditional risk factors. For example, a 2018 simulation study $^{29}$ suggested that lowering nicotine content in cigarettes could result in a $15.6 \%$ decrease in smoking prevalence (from $12.8 \%$ to $10.8 \%$ in the overall US population) in the first year of the policy. The benefit of this structural intervention would not be limited to reducing smoking prevalence among adults with HIV but would also benefit the general population. The evaluation of the effectiveness of modified interventions and cost analyses for effective interventions are needed.

Individual-level traditional risk factor interventions must be accessible to adults with HIV; herein lie the strength of the associations between smoking and substantial challenges. Driven by the increased life expectancy of adults with HIV initiating ART at early stages of disease, the size of the population of adults with HIV will continue to increase within the context of current and projected shortages of HIV care providers in some settings, such as the USA. ${ }^{30}$ There is a need for sustainable models that have the capacity for risk factor interventions (many of which might need to be offered numerous times over decades of life with HIV) and management of complex health-care needs with multidisciplinary teams, when non-communicable diseases are not avoided. General-practitioner, nurse-practitioner, and care-coordinator models that are common in other medically complex settings (such as oncology and transplantation) should be evaluated for implementation as the primary medical home for adults with HIV. More formal adoption of geriatric-medicine principles and patient-centred care approaches in HIV-care models might be particularly beneficial. ${ }^{31-33}$

We caution against common misinterpretation of PAFs, which should be interpreted as the "proportion of disease cases over a specified time that would be prevented following elimination of the exposure, assuming the exposure is causal". ${ }^{34}$ The relationhips between the risk factors included in this investigation and each outcome range from meeting Bradford Hill's causal criteria (eg, smoking and non-AIDS defining cancers) to being incompletely verified as causal (eg, HIV RNA, which is a time-dependent surrogate for inflammation from uncontrolled viraemia). ${ }^{35}$ Although the interpretation of the PAF is one of prevention of the risk factor (ie, the proportion of the outcome that could be avoided if all members of the study population never started smoking), modification of these risk factors might be a more achievable approach than prevention. It is commonly assumed that the proportion of outcomes avoided by modifying the risk factor (eg, smoking cessation) is likely to be similar to that of preventing the risk factor (ie, smoking prevention), although it might be smaller because the risk of developing the outcome does not immediately drop to that of the unexposed group (ie, nonsmokers) at the time of modification (ie, cessation). ${ }^{36}$ However, we did not directly assess the effect of modification.

We also caution against inappropriately summing the single risk factor PAFs in an attempt to derive the total fraction of disease risk attributable to all of the factors because the few conditions under which this approach is valid do not apply to this study. ${ }^{37}$

Our approach might have underestimated PAFs for proportion of outcomes that would be avoided if smoking were prevented was probably underestimated because current and former smokers were combined in the category of participants who have ever smoked, thus increasing the prevalence of this exposure but diluting many of the traditional risk factors. For example, the 
the outcomes. Because hypertension was categorised as diagnosed and treated hypertension, the prevalence was probably underestimated and the associations between hypertension and the outcomes might have been diluted (systolic and diastolic blood pressure measurements were not available for this study). This could also be the case for diabetes, as the individuals with undiagnosed diabetes and no glycated haemoglobin $\mathrm{A}_{1 \mathrm{c}}$ test did not meet the criteria to be classified as having diabetes, thus reducing the prevalence of diabetes and potentially diluting the association between diabetes and the outcomes. The PAF for hepatitis $\mathrm{C}$ infection might also have been affected by the way we defined having a positive antibody, which included individuals who resolved their hepatitis $\mathrm{C}$ infection, and thus might have caused an overestimation of the prevalence of hepatitis $C$ but probably diluted the associations with the outcomes. Direct acting antivirals for hepatitis $\mathrm{C}$ were not licensed until the last year of our study.

Although we examined consistently measured risk factors across four validated non-communicable disease events, a limitation of our study is the possibility that additional preventable or modifiable causal risk factors were not considered, such as specific antiretroviral drugs and regimens (and associated metabolic effects), diet, physical activity, and proteinuria. To estimate the PAF for specific antiretroviral drugs and regimens, methods are needed to account for confounding by indication (perhaps by incorporating weights into the approach) so that specific antiretroviral drugs and regimens do not show spuriously protective outcomes. Furthermore, investigations into causal interactions between specific antiretroviral drugs or regimens and risk factors are needed to incorporate the complexities of HIV treatments when estimating the burden of non-communicable-disease outcomes that could be avoided. Risk factors that are not preventable or modifiable (such as the estimated 66\% of cancer-driving mutations that are caused by random DNA replication errors in healthy, dividing cells) might also have been at play but unaccounted for. ${ }^{38}$ Although we cannot weigh the benefit of intervention upon these omitted risk factors, we believe we have included the most relevant known risk factors for each outcome. Additionally, current smoking was not included as a possible category of smoking, which is an important limitation. Given the high prevalence of smoking and the low cessation rates among adults with HIV, we suspect the PAF for ever smoking presented here is between the unknown PAFs for current and former smoking. ${ }^{27,28}$ Finally, our findings cannot be interpreted as the effect of HIV itself on these outcomes because all patients in this study were infected with HIV; the goal was to assess the population-level effect of traditional and HIVrelated risk factors on non-AIDS-defining cancers, myocardial infarction, end-stage liver disease, and endstage renal disease among adults ageing with HIV.

In summary, preventing the high burden of noncommunicable diseases among adults ageing with HIV will require prioritisation of interventions. Our findings show that individual and structural or policy-level interventions on traditional risk factors in the context of antiretroviral-induced chronic viral suppression could prevent a substantial proportion of non-communicable diseases. Interventions to prevent and address smoking, elevated total cholesterol, and hypertension are particularly important for reducing non-AIDS-defining cancers, myocardial infarction, and end-stage renal disease outcomes. Hepatitis $\mathrm{C}$ infection and at-risk alcohol use prevention and treatment are crucial to reducing the burden of end-stage liver disease. Modifications to individual-level interventions and models of HIV care, and the implementation of structural and policy-level interventions that focus on prevention and modification of traditional risk factors are necessary to avoid non-communicable diseases and preserve health among successfully antiretroviral-treated adults ageing with HIV.

\section{Contributors}

KNA, KAG, ACJ, and CW contributed to literature search. KNA, KAG RDM, CMB, ACJ, CW, GML, MBK, MMK, HC, MJS, MJG, WCM, RD MAH, CSR, DBK, VLR, TRS, FAD, KL, JW, ARR, GDK, KA, FJP Jr, JET, JE, LPJ, SN, CA, AMM, PP, KB, YJ, and SJG contributed to study design and data interpretation. KAG, RDM, ACJ, GML, MBK, MMK, HC, MJS MJG, WCM, RD, MAH, CSR, DBK, VLR, TRS, KL, JW, ARR, GDK, KA, FJP Jr, JET, JE, LPJ, SN, CA, AMM, PP, KB, and SJG contributed to data collection. KNA, YJ, and SJG contributed to data analysis. KNA wrote the initial draft of the manuscript and created the tables and figures. All authors reviewed the manuscript, provided substantive feedback, and approved submission.

\section{Declaration of interests}

KNA, RDM, ACJ, GML, MMK, HC, MJS, MJG, RD, VLR, TRS, FAD, JW GDK, KA, FJP, JET, JE, LPJ, SN, CA, and SJG report grants from the National Institutes of Health during the conduct of the study and outside the submitted work. KNA also reports personal fees from Trio Health outside of the submitted work. KAG reports grants from Agency for Healthcare Research and Quality (AHRQ; USA) and Health Resources and Services Administration (HRSA; USA) during the conduct of the study, and personal fees from the US Government outside the submitted work. RDM also reports grants from AHRQ and HRSA during the conduct of the study, and personal fees from Medscape LLC outside the submitted work. CMB reports royalties from Up To Date outside of the submitted work. MBK reports funding from the Canadian Institutes of Health Research (CIHR), The CIHR Canadian HIV Trials Network, and le Réseau sida et maladies infectieuses du Fonds de rescherche Santé Québec, for investigator-initiated trials from ViiV Healthcare and Gilead Sciences and personal fees from ViiV Healthcare and Merck, outside the submitted work. HC also reports personal fees from ViiV outside the submitted work. MJS also reports grants from Merck and Gilead outside the submitted work. MJG also reports personal fees from Merck, ViiV Healthcare, and Gilead outside the submitted work. ARR reports grants from Ontario HIV Treatment Network during the conduct of the study, and grants from Merck Frostt, ViiV Healthcare, Gilead Sciences, and Janssen outside the submitted work. FJP also reports personal fees from Merck, ViiV Healthcare, Gilead Sciences, and Janssen, outside the submitted work. JET also reports grants from Allergan and personal fees from AbbVie, Santen, and Gilead outside the submitted work. MAH reports grants from Merck and Gilead outside the submitted work. JE also reports grants from Janssen, ViiV Healthcare, Gilead Sciences, and personal fees from Janssen, Gilead Sciences, ViiV Healthcare, and Merck outside the submitted work. CA also reports grants Gilead and personal fees from ViiV outside of the submitted work. CW, WCM, DBK, CSR, KL, AMM $\mathrm{PP}, \mathrm{KB}$, and $\mathrm{YJ}$ have nothing to disclose. 


\section{Acknowledgments}

The content is solely the responsibility of the authors and does not necessarily represent the official views of the National Institutes of Health or the US Centers for Disease Control and Prevention. KNA was supported by K01AI093197, National Institute of Allergy and Infectious Diseases, National Institutes of Health. Additionally, this work was supported by National Institutes of Health grants U01AI069918, F31AI124794, F31DA037788, G12MD007583, K01AI093197, K23EY013707, K24AI065298, K24AI118591, K24DA000432, K24DA035684,

KL2TR000421, M01RR000052, N01CP01004, N02CP055504, N02CP91027 P30AI027757, P30AI027763, P30AI027767, P30AI036219, P30AI050410, P30AI094189, P30AI110527, P30MH62246, R01AA016893, R01CA165937, R01DA011602, R01DA012568, R01AG053100, R01DA026770, R24AI067039, R24AG044325, U01AA013566, U01AA020790, U01AI031834, U01AI034989, U01AI034993, U01AI034994, U01AI035004, U01AI035039, U01AI035040, U01AI035041, U01AI035042, U01AI037613, U01AI037984, U01AI038855, U01AI038858, U01AI042590,

U01AI068634, U01AI068636, U01AI069432, U01AI069434, U01AI103390, U01AI103397, U01AI103401, U01AI103408, U01DA03629, U01DA036935, U01HD032632, U10EY008057, U10EY008052, U10EY008067, U24AA020794, U54MD007587, UL1RR024131, UL1TR000004, UL1TR000083, UL1TR000454, UM1AI035043, Z01CP010214, and Z01CP010176; contracts CDC-200-2006-18797 and CDC-200-2015-63931 from the US Centers for Disease Control and Prevention; contract 90047713 from the AHRQ; contract 90051652 from the HRSA; grants CBR-86906, CBR-94036, HCP-97105 and TGF-96118 from the Canadian Institutes of Health Research, Canada; Ontario Ministry of Health and Long Term Care; and the Government of Alberta, Canada. Additional support was provided by the National Cancer Institute, National Institute for Mental Health, National Institute on Drug Abuse, the Johns Hopkins Center for AIDS Research (P30 AI094189), and the Sidney Kimmel Comprehensive Cancer Center research programme grant (P30 CA006973).

\section{NA-ACCORD Collaborating Cohorts and Representatives}

AIDS Clinical Trials Group Longitudinal Linked Randomized Trials: Constance A Benson and Ronald J Bosch; AIDS Link to the IntraVenous Experience: Gregory D Kirk; Fenway Health HIV Cohort: Stephen Boswell, Kenneth H Mayer and Chris Grasso; HAART Observational Medical Evaluation and Research: Robert S Hogg, P Richard Harrigan, Julio S G Montaner, Benita Yip, Julia Zhu, Kate Salters and Karyn Gabler; HIV Outpatient Study: Kate Buchacz and John T Brooks; HIV Research Network: Kelly A Gebo and Richard D Moore; Johns Hopkins HIV Clinical Cohort: Richard D Moore; John T Carey Special Immunology Unit Patient Care and Research Database, Case Western Reserve University: Benigno Rodriguez; Kaiser Permanente Mid-Atlantic States: Michael A Horberg; Kaiser Permanente Northern California: Michael J Silverberg; Longitudinal Study of Ocular Complications of AIDS: Jennifer E Thorne; Multicenter Hemophilia Cohort Study-II: Charles Rabkin; Multicenter AIDS Cohort Study: Joseph B Margolick, Lisa P Jacobson, and Gypsyamber D’Souza; Chronic Viral Illness Service Cohort of the McGill University Health Centre: Marina B Klein; Ontario HIV Treatment Network Cohort Study: Sean B Rourke, Anita R Rachlis, and Patrick Cupido; Retrovirus Research Center, Bayamon Puerto Rico: Robert F Hunter-Mellado and Angel M Mayor; Southern Alberta Clinic Cohort: M John Gill; Study of the Consequences of the Protease Inhibitor Era: Steven G Deeks and Jeffrey N Martin; Study to Understand the Natural History of HIV/AIDS in the Era of Effective Therapy: Pragna Patel and John T Brooks; University of Alabama at Birmingham 1917 Clinic Cohort: Michael S Saag, Michael J Mugavero, and James Willig; University of California at San Diego: William C Mathews; University of North Carolina at Chapel Hill HIV Clinic Cohort: Joseph J Eron and Sonia Napravnik; University of Washington HIV Cohort: Mari M Kitahata, Heidi M Crane, and Daniel R Drozd; Vanderbilt Comprehensive Care Clinic HIV Cohort: Timothy R Sterling, David Haas, Peter Rebeiro, Megan Turner, Sally Bebawy, and Ben Rogers; Veterans Aging Cohort Study: Amy C Justice, Robert Dubrow, and David Fiellin; Women's Interagency HIV Study: Stephen J Gange and Kathryn Anastos; NA-ACCORD Study Administration; Executive Committee: Richard D Moore, Michael S Saag, Stephen J Gange, Mari M Kitahata, Keri N Althoff,
Michael A Horberg, Marina B Klein, Rosemary G McKaig, and Aimee M Freeman; Administrative Core: Richard D Moore, Aimee M Freeman, and Carol Lent; Data Management Core: Mari M Kitahata, Stephen E Van Rompaey, Heidi M Crane, Daniel R Drozd, Liz Morton, Justin McReynolds, and William B Lober; Epidemiology and Biostatistics Core: Stephen J Gange, Keri N Althoff, Jennifer Lee, Bin You, Brenna Hogan, Elizabeth Humes, Jinbing Zhang, Jerry Jing, Bin Liu, and Fidel A Desir.

\section{References}

1 Althoff K, Smit M, Reiss P, Justice AC. HIV and ageing: improving quantity and quality of life. Curr Opin HIV AIDS 2016; 11: $527-36$.

2 Park LS, Hernández-Ramírez RU, Silverberg MJ, Crothers K, Dubrow R. Prevalence of non-HIV cancer risk factors in persons living with HIV/AIDS: a meta-analysis. AIDS 2016; 30: 273-91.

3 Friis-Møller N, Weber R, Reiss P, et al. Cardiovascular disease risk factors in HIV patients-association with antiretroviral therapy. Results from the DAD study. AIDS 2003; 17: 1179-93.

4 Sulkowski MS, Moore RD, Mehta SH, Chaisson RE, Thomas DL. Hepatitis C and progression of HIV disease. JAMA 2002; 288: 199-206.

5 Deeks SG. HIV infection, inflammation, immunosenescence, and aging. Annu Rev Med 2011; 62: 141-55.

6 Wada NI, Jacobson LP, Margolick JB, et al. The effect of HAART-induced HIV suppression on circulating markers of inflammation and immune activation. AIDS 2015; 29: 463-71.

7 Friis-Møller N, Smieja M, Klein D. Antiretroviral therapy as a cardiovascular disease risk factor: fact or fiction? A review of clinical and surrogate outcome studies. Curr Opin HIV AIDS 2008; 3: 220-25.

8 Borges ÁH, Neuhaus J, Babiker AG, et al. Immediate antiretroviral therapy reduces risk of infection-related cancer during early HIV infection. Clin Infect Dis 2016; 63: 1668-76.

9 Smith CJ, Ryom L, Weber R, et al. Trends in underlying causes of death in people with HIV from 1999 to 2011 (D:A:D): a multicohort collaboration. Lancet 2014; 384: 241-48.

10 Weber R, Ruppik M, Rickenbach M, et al. Decreasing mortality and changing patterns of causes of death in the Swiss HIV Cohort Study. HIV Med 2013; 14: 195-207.

11 Klein MB, Rollet-Kurhajec KC, Moodie EEM, et al. Mortality in HIV-hepatitis C co-infected patients in Canada compared to the general Canadian population (2003-2013). AIDS 2014; 28: 1957-65.

12 Platt L, Easterbrook P, Gower E, et al. Prevalence and burden of HCV co-infection in people living with HIV: a global systematic review and meta-analysis. Lancet Infect Dis 2016; 16: 797-808.

13 Sun H-Y, Sheng W-H, Tsai M-S, et al. Hepatitis B virus coinfection in human immunodeficiency virus-infected patients: a review. World J Gastroenterol 2014; 20: 14598-614.

14 Abraham AG, Althoff KN, Jing Y, et al. End-stage renal disease among HIV-infected adults in North America. Clin Infect Dis 2015; 60: 941-49.

15 Mocroft A, Lundgren JD, Ross M, et al. Cumulative and current exposure to potentially nephrotoxic antiretrovirals and development of chronic kidney disease in HIV-positive individuals with a normal baseline estimated glomerular filtration rate: a prospective international cohort study. Lancet HIV 2016; 3: 23-32.

16 Gange SJ, Kitahata MM, Saag MS, et al. Cohort profile: the North American AIDS Cohort Collaboration on Research and Design (NA-ACCORD). Int J Epidemiol 2007; 36: 294-301.

17 Silverberg MJ, Lau B, Achenbach CJ, et al. Cumulative incidence of cancer among persons with HIV in North America: a cohort study. Ann Intern Med 2015; 163: 507-18.

18 Crane HM, Heckbert SR, Drozd DR, et al. Lessons learned from the design and implementation of myocardial infarction adjudication tailored for HIV clinical cohorts. Am J Epidemiol 2014; 179: 996-1005.

19 Kitahata MM, Drozd DR, Crane HM, et al. Ascertainment and verification of end-stage renal disease and end-stage liver disease in the North American AIDS Cohort Collaboration on Research and Design. AIDS Res Treat; 2015: 923194.

20 Levey AS, Stevens LA, Schmid CH, et al. A new equation to estimate glomerular filtration rate. Ann Intern Med 2009; 150: 604-12 
21 Castro KG, Ward JW, Slutsker L, et al. 1993 Revised classification system for HIV infection and expanded surveillance case definition for AIDS among adolescents and adults. MMWR 1992; 41: 1-19.

22 Laaksonen MA, Virtala E, Knekt P, Oja H, Härkänen T. SAS macros for calculation of population attributable fraction in a cohort study design. J Stat Softw 2011; 43: 1-25.

23 Hiller L, Marshall A, Dunn J. Assessing violations of the proportional hazards assumption in Cox regression: does the chosen method matter? Trials 2015; 16: P134.

24 Tsimihodimos V, Mitrogianni Z, Elisaf M. Dyslipidemia associated with chronic kidney disease. Open Cardiovasc Med J 2011; 5: 41-48.

25 Althoff KN. The shifting paradigm of care for adults living with HIV: smoking cessation for longer life. J Infect Dis 2016; 214: 1618-20.

26 Mercié P, Arsandaux J, Katlama C, et al. Efficacy and safety of varenicline for smoking cessation in people living with HIV in France (ANRS 144 Inter-ACTIV): a randomised controlled phase 3 clinical trial. Lancet HIV 2018; 5: 126-35.

27 Mdodo R, Frazier EL, Dube SR, et al. Cigarette smoking prevalence among adults with HIV compared with the general adult population in the United States: cross-sectional surveys. Ann Intern Med 2015; 162: 335-44.

28 Hessol NA, Weber KM, D’Souza G, et al. Smoking cessation and recidivism in the Women's Interagency Human Immunodeficiency Virus Study. Am J Prev Med 2014; 47: 53-69.

29 Apelberg BJ, Feirman SP, Salazar E, et al. Potential public health effects of reducing nicotine levels in cigarettes in the United States. N Engl J Med 2018; 378: 1725-33.
30 Walensky RP, Del Rio C, Armstrong WS. Charting the future of infectious disease: anticipating and addressing the supply and demand mismatch. Clin Infect Dis 2017; 64: 1299-301.

31 Boyd CM, Lucas GM. Patient-centered care for people living with multimorbidity. Curr Opin HIV AIDS 2014; 9: 419-27.

32 Singh HK, Del Carmen T, Freeman R, Glesby MJ, Siegler EL. From one syndrome to many: incorporating geriatric consultation into HIV care. Clin Infect Dis 2017; 65: 501-06.

33 Guaraldi G, Rockwood K. Geriatric-HIV medicine is born. Clin Infect Dis 2017; 65: 507-09.

34 Rockhill B, Newman B, Weinberg C. Use and misuse of population attributable fractions. Am J Pub Health 1998; 88: 15-19.

35 Hill AB. The environment and disease: association or causation? Proc R Soc Med 1965; 58: 295-300.

36 Peto R, Darby S, Deo H, Silcocks P, Whitley E, Doll R. Smoking, smoking cessation, and lung cancer in the UK since 1950: combination of national statistics with two case-control studies. BMJ 2000; 321: 323-29.

37 Walter SD. The estimation and interpretation of attributable risk in health research. Biometrics 1976; 32: 829-49.

38 Tomasetti C, Li L, Vogelstein B. Stem cell divisions, somatic mutations, cancer etiology, and cancer prevention. Science 2017; 355: 1330-34. 